\title{
Elaboração e Validação de um Programa de Exercícios para Mulheres Submetidas à Cirurgia Oncológica de Mama
}

\author{
Development and Validation of an Exercise Program for Women Submitted to \\ Breast Cancer Surgery
}

\author{
Eliana Louzada Petito', Maria Gaby Rivero de Gutiérrez²
}

\begin{abstract}
Resumo
Introdução: $\mathrm{O}$ benefício da reabilitação funcional precoce em mulheres submetidas à cirurgia oncológica de mama é amplamente reconhecido. Porém não existe consenso sobre quais os exercícios mais indicados, a periodicidade da realização dos mesmos e a duração do programa. Objetivo: Identificar e selecionar um conjunto de exercícios para compor um programa de reabilitação funcional pós-operatória de cirurgia oncológica de mama visando à recuperação funcional do membro homolateral à cirurgia e submetê-lo à validação com especialistas. Método: Revisão bibliográfica de estudos que testassem a efetividade de programas de reabilitação funcional da amplitude de movimento do ombro em cirurgia oncológica de mama e publicados entre 1997 e 2006 nas bases de dados MEDLINE, LILACS e PUBMED. Seleção de um conjunto de exercícios para recuperação da amplitude de movimento do ombro, e submissão dos mesmos à avaliação por especialistas em reabilitação. Resultados: Dos 21 artigos identificados, 12 se referiam ao teste ou padronização de procedimentos para reabilitação funcional pósoperatória, os quais constituíram a base para a seleção dos 20 exercícios submetidos à avaliação dos especialistas. Desse total, nove foram por elas considerados indispensáveis, com um índice de concordância igual ou superior a 75\%. Os exercícios que obtiveram maior índice de concordância foram os de alongamento cervical, flexão, extensão e abdução do ombro. Conclusão: Os resultados deste estudo contribuíram para a reformulação do manual para Reabilitação Funcional Precoce de Mulheres Mastectomizadas do Setor de Oncomastologia da Disciplina de Mastologia da UNIFESP.

Palavras-chave: Exercício; Mastectomia; Neoplasias mamárias; Fisioterapia (especialidades); Enfermagem oncológica
\end{abstract}

Instituição: Escola de Enfermagem - Universidade Federal de São Paulo (UNIFESP)

Agência de fomento: CNPq - processo 473174/2004-9

${ }^{1}$ Fisioterapeuta, mestranda do Programa de Pós-Graduação em Enfermagem da Universidade Federal de São Paulo (UNIFESP)

${ }^{2}$ Professora associada da Escola de Enfermagem da UNIFESP

Endereço para correspondência: Eliana Louzada Petito. Rua Capote Valente, 929 - ap 43A - São Paulo (SP), Brasil - CEP: $05409-002$.

E-mail: lica.sp@ig.com.br 


\section{INTRODUÇÃO}

A cirurgia é um dos tratamentos de escolha para o câncer de mama e, mesmo em face aos avanços tecnológicos, existe a possibilidade de que esse tipo de intervenção cirúrgica possa trazer complicaçōes, tais como: linfedema, alteração postural, limitação da amplitude de movimento (ADM), perda ou diminuição da função e dor no membro homolateral após a cirurgia ${ }^{1-10}$.

A literatura disponível sobre o assunto é unânime em destacar a importância da realização de exercícios físicos a fim de prevenir essas complicações pósoperatórias, porém não existe consenso sobre quais exercícios são os mais indicados, a periodicidade da realização dos mesmos, e a duração do programa ${ }^{1-7}$.

Dentre os 12 artigos selecionados na revisão de literatura, dez enfatizavam a importância da avaliação fisioterápica pré-operatóriaa ${ }^{2,4,6,11,12}$, ou no $1^{\circ}$ pósoperatório (PO) $)^{1,3,5,13,14}$. Todos os programas descritos nos 12 artigos incluem a realização dos movimentos de flexão, extensão, abdução e adução dos ombros, visando à recuperação da ADM. Cabe destacar que a inclusão de exercícios para fortalecimento muscular foi citada apenas em três trabalhos ${ }^{3,5,14}$, com a utilização de bolinhas, exercícios isométricos em fase inicial do programa, ou uso de faixas elásticas, a partir da $4^{\mathrm{a}}-6^{\mathrm{a}}$ semana.

Diante do exposto, considerou-se necessário reavaliar o programa para reabilitação pós-operatória de mulheres mastectomizadas, implementado no Setor de Oncomastologia da Disciplina de Mastologia da Universidade Federal de São Paulo - UNIFESP.

Para tanto, decidiu-se realizar uma revisão de literatura com os seguintes objetivos: identificar e selecionar um conjunto de exercícios para compor um programa de reabilitação funcional precoce de mulheres mastectomizadas e submetê-lo à avaliação de especialistas.

\section{MÉTODO}

Para identificação e seleção dos exercícios para avaliação dos especialistas, foi realizada uma revisão bibliográfica nas bases de dados MEDLINE, LILACS e PUBMED, utilizando os descritores DeCS/MeSH: neoplasia de mama, exercícios, reabilitação, fisioterapia, mastectomia, incluindo artigos publicados entre 1997 e 2006, nos idiomas português, inglês e espanhol.

Os artigos selecionados foram aqueles que descreviam programas de exercícios pós-operatórios para mulheres submetidas à cirurgia de câncer de mama, seja quadrantectomia ou mastectomia, com ou sem linfadenectomia, porém sem reconstrução mamária conjunta, bem como manuais de Instituiçôes Brasileiras de referência para tratamento e reabilitação de pacientes com câncer. Os aspectos levantados foram: avaliação da ADM, exercícios propostos e periodicidade da realização dos mesmos.

Após essa fase, foram selecionados 20 exercícios, agrupados de acordo com o tipo de movimento: três para alongamento da região cervical, três para movimento da cintura escapular, quatro para flexão dos ombros, três para extensão dos ombros, cinco para adução e abdução dos ombros e dois para fortalecimento, pois quatro artigos incluíam essa modalidade de exercícios em seus programas, quer seja com isometria do ombro, uso de bolinhas ou resistência leve $3,5,12,14$ (Anexo 1).

Os 20 exercícios selecionados, citados com maior freqüência nos artigos revisados, foram submetidos à avaliação de um médico mastologista, uma enfermeira e seis fisioterapeutas, todos com atuação na área de Mastologia, com a finalidade de que os classificassem em indispensáveis, secundários ou desnecessários para compor um programa de exercícios para mulheres submetidas à cirurgia oncológica de mama.

Os exercícios que apresentaram um índice de concordância igual ou superior a $75 \%$ na classificação como indispensáveis foram utilizados para reformular o manual existente no Setor de Oncomastologia da Disciplina de Mastologia da UNIFESP que atende a mulheres usuárias do Sistema Único de Saúde (SUS). Os resultados foram analisados de forma descritiva e serão expostos em tabelas e figuras.

\section{RESULTADOS}

$\mathrm{Na}$ revisão bibliográfica, foram encontrados 22 artigos sobre cirurgia oncológica de mama e exercícios físicos, dos quais nove referiam-se a estudos que testavam programas de exercícios e três descreviam a padronização de condutas para programas de exercícios pós-operatória de câncer de mama. Os dez restantes não foram incluídos nas tabelas por abordarem aspectos gerais da cirurgia de mama, não apresentando a descrição dos programas de exercícios. Os resultados obtidos estão descritos nas tabelas 1 e 2 .

A tabela 1 apresenta nove estudos prospectivos de intervenção e seguimento por diferentes períodos: 1 mês $^{7}, 6$ semanas $^{6,12,14}, 2$ meses ${ }^{1}, 3$ meses $^{4}, 12$ meses $^{11}$, 24 meses $^{2}$. Todos propunham um programa de exercícios para recuperação da ADM do ombro homolateral à cirurgia e de prevenção da formação de linfedema, por meio da realização diária e progressiva de diferentes exercícios, a partir do $1^{\circ} \mathrm{PO}$, enfatizando os movimentos de alongamento e relaxamento da região cervical e cintura escapular, flexão e abdução do ombro, sendo que apenas quatro trabalhos destacavam exercícios para a extensão do ombro ${ }^{1,6,11,13}$.

O programa pós-operatório incluía a realização de exercícios no período de internação e após a 
alta hospitalar, em sessões semanais no bem como sua realização no domicílio, após orientação ambulatório, sob a orientação de um profissional, e entrega de manual ${ }^{1,2,4,6,7,11-12,15}$.

Tabela 1. Estudos experimentais sobre programas de exercícios para mulheres submetidas à cirurgia por câncer de mama (1997 - 2006)

\begin{tabular}{|c|c|c|c|c|c|c|}
\hline \multirow{2}{*}{ AUTOR } & \multirow{2}{*}{ MÉTODO } & \multicolumn{5}{|c|}{ INTERVENÇÃO } \\
\hline & & TRATAMENTO & POPULAÇÃO & DURAÇÃO & PROTOCOLO & RESULTADOS \\
\hline $\begin{array}{l}\text { Box RC } \\
\text { ef al. }{ }^{2}\end{array}$ & $\begin{array}{l}\text { randomizado } \\
\text { parcialmente } \\
\text { cego }\end{array}$ & $\begin{array}{l}\text { Cirurgia } \\
\text { Conservadora } \\
\text { C/ EA ou } \\
\text { MRM }\end{array}$ & $\begin{array}{l}33 G C \\
32 G E\end{array}$ & $\begin{array}{l}\text { - Av: pré-op., } \\
\text { até } 24 \\
\text { meses } \\
\text { - Início dos } \\
\text { exercícios: } \\
2^{\circ} \text { PO }\end{array}$ & $\begin{array}{l}\text { - GE - FST } \\
\text { - GC - manual de } \\
\text { exercícios } \\
\text { - progressão gradual dos } \\
\text { exercícios }\end{array}$ & $\begin{array}{l}\text { - FST: eficiente p/ recuperar a } \\
\text { ADM do ombro em } 2 \text { anos } \\
\text { - programa otimiza a } \\
\text { recuperação da ADM }\end{array}$ \\
\hline $\begin{array}{c}\text { Marimoto } \\
\text { T et al. }\end{array}$ & $\begin{array}{c}\text { não } \\
\text { controlado }\end{array}$ & $\begin{array}{l}\text { MRM ou } \\
\text { cirurgia } \\
\text { Conservadora }\end{array}$ & 72 & $\begin{array}{l}\text { - Av: pré-op., } \\
\text { até } 12 \\
\text { semanas } \\
\text { - Início dos } \\
\text { exercícios: } \\
1^{\circ}-4^{\circ} \mathrm{PO}\end{array}$ & $\begin{array}{l}\text { - manual + vídeo + } \\
\text { exercícios } \\
\text { - progressão gradual dos } \\
\text { exercícios }\end{array}$ & $\begin{array}{l}\text { - recuperação da ADM } \cong 12 \\
\text { Semanas } \\
\text { - } 90 \% \text { pacientes capazes de } \\
\text { realizar AVD }\end{array}$ \\
\hline $\begin{array}{c}\text { Pereira } \\
\text { CM ef al. }^{7}\end{array}$ & $\begin{array}{c}\text { não } \\
\text { controlado }\end{array}$ & $\begin{array}{l}\text { MRM + } 28 \\
\text { sessões de } \\
\quad \text { RXT }\end{array}$ & $\begin{array}{l}11 G C \\
33 G E\end{array}$ & $\begin{array}{l}\text { - Av: } 7^{\circ} \mathrm{PO} \text {, } \\
\text { até } 1 \text { mês } \\
\text { - Início dos } \\
\text { exercícios: } \\
7^{\circ} \mathrm{PO}\end{array}$ & $\begin{array}{l}\text { - GE - pacientes c/ FST até } \\
\text { alta } \\
\text { - GC - pacientes que } \\
\text { desistiram } \\
\text { - orientação + manual + } \\
\text { FST } \\
\text { - progressão gradual dos } \\
\text { exercícios } \\
\text { - limite da paciente }\end{array}$ & $\begin{array}{l}\text { - melhora ADM: GE } 173^{\circ}(\mathrm{fl}) \text {, } \\
174^{\circ} \text { (abd) X GC } 127^{\circ}(\mathrm{fl}) \text {, } \\
120^{\circ} \text { (abd) } \\
\text { - maior incidência de } \\
\text { linfedema: no GC }\end{array}$ \\
\hline $\begin{array}{l}\text { Silva MPP } \\
\text { ef al. }{ }^{6}\end{array}$ & randomizado & $\begin{array}{c}\text { Cirurgia } \\
\text { Conservadora } \\
\text { Ou MRM } \\
+ \\
\text { EA }\end{array}$ & $\begin{array}{l}29 G C \\
30 G E\end{array}$ & $\begin{array}{l}\text { - Av: pré-op., } \\
\text { até } 42^{\circ} \text { PO } \\
\text { - Início dos } \\
\text { exercícios: } \\
1^{\circ} \text { PO }\end{array}$ & $\begin{array}{l}\text { - GE - ADM livre } \\
\text { - } G C \text { - ADM até } 90^{\circ} \\
\text { - progressão gradual dos } \\
\text { exercícios }\end{array}$ & $\begin{array}{l}\text { - GE: boa recuperação da } \\
\text { capacidade funcional do } \\
\text { ombro, sem aumento da } \\
\text { incidência de complicações } \\
\text { - Limitação } \leq 30^{\circ} \text {, compatível } \\
\text { com AVD }\end{array}$ \\
\hline $\begin{array}{l}\text { Sasaki T } \\
\text { ef al. }{ }^{1}\end{array}$ & $\begin{array}{c}\text { não } \\
\text { controlado }\end{array}$ & mastectomia & 13 & $\begin{array}{l}\text { - Av: } 1^{\circ} \mathrm{PO} \text {, } \\
\text { até } 60^{\circ} \mathrm{PO} \\
\text { - Início dos } \\
\text { exercícios: } \\
1^{\circ} \mathrm{PO}\end{array}$ & $\begin{array}{l}\text { - orientação: manual + } \\
\text { FST } \\
\text { - grupo de orientação } \\
\text { multidisciplinar semanal } \\
\text { - progressão gradual dos } \\
\text { exercícios }\end{array}$ & $\begin{array}{l}\text { recuperação total da ADM } \\
\text { entre } 15^{\circ} \text { e } 20^{\circ} \text { PO, sem } \\
\text { prejuízo da cicatrização } \\
\text { - retorno AVD }\end{array}$ \\
\hline $\begin{array}{l}\text { Rezende } \\
\text { LF et al. }{ }^{14}\end{array}$ & randomizado & $\begin{array}{l}\text { MRM ou } \\
\text { cirurgia } \\
\text { Conservadora } \\
+ \text { EA }\end{array}$ & $\begin{array}{l}30 G C \\
30 G E\end{array}$ & $\begin{array}{l}\text { - Av e início } \\
\text { dos } \\
\text { exercícios: } \\
1^{\circ} \text { P0, até } 6 \\
\text { semanas }\end{array}$ & $\begin{array}{l}\text { - GE - FST orientada, com } \\
\text { exercícios pré- } \\
\text { estabelecidos } \\
\text { - GC - exercícios sem } \\
\text { prévia seqüência, ritmo } \\
\text { da música } \\
\text { - progressão gradual dos } \\
\text { exercícios, limite pela } \\
\text { dor ou desconforto }\end{array}$ & $\begin{array}{l}\text { - GE demonstrou maior } \\
\text { eficiência na recuperação da } \\
\text { ADM - } \mathrm{fl} \text {, e abd } \\
\text { - não houve } \neq \text { nos distúrbios } \\
\text { linfáticos em ambos os } \\
\text { grupos }\end{array}$ \\
\hline $\begin{array}{l}\text { Lauridsen } \\
\text { MC et al. } \\
\quad 11\end{array}$ & $\begin{array}{l}\text { randomizado } \\
\text { não-cego }\end{array}$ & $\begin{array}{c}\text { Cirurgia } \\
\text { Conservadora } \\
+ \text { EA + } \\
\text { RXT ou MRM } \\
\text { + EA }\end{array}$ & $\begin{array}{l}72 \mathrm{GC} \\
67 \mathrm{GE}\end{array}$ & $\begin{array}{l}\text { - Av: pré-op., } \\
\text { até } 56 \\
\text { semanas } \\
\text { - Início entre } \\
6^{a} \text { e } 26^{a} \\
\text { semana PO }\end{array}$ & $\begin{array}{l}\text { - GE: FST após } 26^{a} \\
\text { semana PO } \\
\text { - GC: FST na } 6^{a}-8^{a} \\
\text { semana PO } \\
\text { - terapia em grupo + } \\
\text { exercícios em casa } \\
\text { - não excluía paciente por } \\
\text { falta sessões }\end{array}$ & $\begin{array}{l}\text { melhora significativa na } \\
\text { ADM do ombro nas semanas } \\
12 \text { e } 26 \text { de PO com FST }\end{array}$ \\
\hline
\end{tabular}


Tabela 1. (cont.)

\begin{tabular}{|c|c|c|c|c|c|c|}
\hline \multirow{2}{*}{ AUTOR } & \multirow{2}{*}{ MÉTODO } & \multicolumn{5}{|c|}{ INTERVENÇÃO } \\
\hline & & TRATAMENTO & POPULAÇÃO & DURAÇÃO & PROTOCOLO & RESULTADOS \\
\hline $\begin{array}{c}\text { Na YM et } \\
\text { al. }^{12}\end{array}$ & randomizado & $M R M+E A$ & $\begin{array}{l}13 G C \\
20 G E\end{array}$ & $\begin{array}{l}\text { - Av: pré-op., } \\
\text { até } 1 \text { mês } \\
\text { - Início dos } \\
\text { exercícios: } \\
1^{\circ} \text { PO }\end{array}$ & $\begin{array}{l}\text { - GE - FST 1'PO + } \\
\text { manual } \\
\text { - } \mathrm{GC} \text { - instruções + } \\
\text { manual }\end{array}$ & $\begin{array}{l}\text { - } \text { estatisticamente } \\
\text { significativa na ADM de fl e } \\
\text { abd após } 1 \text { mês da alta } \\
\text { hospitalar }\end{array}$ \\
\hline $\begin{array}{l}\text { Leal TO } \\
\text { ef al. }{ }^{15}\end{array}$ & randomizado & $\begin{array}{l}\text { MRM + } \\
\text { EA+ QT e } \\
\quad \text { RXT }\end{array}$ & $\begin{array}{l}10 G C \\
10 G E\end{array}$ & $\begin{array}{l}\text { - Não } \\
\text { específica }\end{array}$ & $\begin{array}{l}\text { - } 2 \text { grupos: FST } \\
\text { lx/semana, mínimo } 10 \\
\text { sessões (GE), s/ FST (GC) } \\
\text { - medidas ADM, força } \\
\text { muscular }\end{array}$ & $\begin{array}{l}\text { f estatisticamente } \\
\text { significativa ADM: fl e abd } \\
\text { do ombro }\end{array}$ \\
\hline
\end{tabular}

$\mathrm{EA}=$ esvaziamento axilar; $\mathrm{MRM}=$ mastectomia radical modificada; $\mathrm{Av}=$ avaliação; pré-op.= pré-operatório; $\mathrm{PO}=$ pós-operatório; $\mathrm{ADM}=$ amplitude de movimento; $\mathrm{GE}=$ grupo experimental; $\mathrm{GC}=$ grupo controle; $\mathrm{FST}=$ fisioterapia; $\mathrm{AVD}=$ atividades de vida diária; $\mathrm{fl}=$ flexão; ext.= extensão; abd= abdução; $\mathrm{RXT}=$ radioterapia; $\mathrm{QT}=$ quimioterapia.

Os trabalhos descritivos estão apresentados na tabela 2 e descrevem a padronização do atendimento fisioterápico nas respectivas instituiçōes ${ }^{3,5,12}$.

Todos os trabalhos mencionados enfatizam que um programa de exercícios supervisionado traz inúmeros benefícios às mulheres submetidas à cirurgia para retirada de câncer de mama, sendo eficientes na recuperação mais rápida da $\mathrm{ADM}$ do ombro, e a maioria ${ }^{2,4,6,14,11}$ incluiu, em sua população, mulheres submetidas a diferentes tipos de procedimentos cirúrgicos (cirurgia conservadora ou mastectomia, com ou sem esvaziamento axilar), bem como à radioterapia e quimioterapia adjuvante.
No que diz respeito à avaliação dos especialistas, será apresentada inicialmente a caracterização das mesmas e, a seguir, a classificação dos exercícios por eles realizados.

Os oito especialistas consultados atuam em instituições conceituadas na área de oncologia, com tempo de formação em medicina, fisioterapia ou enfermagem entre 2 e 23 anos, possuem especialização na área de mastologia (3), mestrado (2), doutorado (3), e atuação na área de Oncomastologia entre 1 e 14 anos, sendo sete do sexo feminino.

No que se refere ao resultado da classificação dos 20 exercícios selecionados e submetidos à

Tabela 2. Estudos descritivos sobre programas de exercícios para mulheres submetidas à cirurgia por câncer de mama (1997 - 2006)

\begin{tabular}{|c|c|c|c|c|c|c|}
\hline \multirow{2}{*}{ AUTOR } & \multirow{2}{*}{ MÉTODO } & \multicolumn{5}{|c|}{ INTERVENÇÃO } \\
\hline & & POPULAÇÃOO & DURAÇÃOO & PROGRAMA & PROTOCOLO & RESULTADOS \\
\hline $\begin{array}{c}\text { Bruno AA } \\
\text { et al. }\end{array}$ & $\begin{array}{l}\text { padronização } \\
\text { de condutas }\end{array}$ & - & - & - $1^{\circ} \mathrm{PO}$ & $\begin{array}{l}\text { - progressão gradual dos } \\
\text { exercícios } \\
\text { - orientações para evitar } \\
\text { complicações tardias }\end{array}$ & $\begin{array}{l}\text { - garantir rápida } \\
\text { integraçáo psicofísica } \\
\text { para retorno à vida } \\
\text { diária }\end{array}$ \\
\hline $\begin{array}{l}\text { Bergmann } \\
\text { A ef al. }{ }^{13}\end{array}$ & $\begin{array}{l}\text { padronização } \\
\text { de condutas }\end{array}$ & - & $\begin{array}{c}\text { Pré-op., } \\
1^{\circ} \mathrm{PO}, 30^{\circ} \mathrm{e} \\
180^{\circ} \text { dias } \mathrm{PO}\end{array}$ & - $1^{\circ} \mathrm{PO}$ & $\begin{array}{l}\text { - } A D M=90^{\circ} \text { até retirada } \\
\text { dos pontos } \\
\text { - retorno gradativo AVD } \\
\text { - atendimentos } \\
\text { fisioterápicos quando } \\
\text { necessários, entre as } \\
\text { reavaliações de } \\
\text { seguimento e/ou } \\
\text { internações clínicas }\end{array}$ & $\begin{array}{l}\text { - implantação de rotina } \\
\text { de atendimento - } \\
\text { prevenção de } \\
\text { complicações, } \\
\text { diagnóstico e } \\
\text { intervenção precoce }\end{array}$ \\
\hline $\begin{array}{l}\text { McAnaw } \\
\text { MB ef al. } .^{5}\end{array}$ & $\begin{array}{c}\text { relato } \\
\text { experiência } \\
\text { local }\end{array}$ & $\begin{array}{c}\text { MRM e MRM } \\
\text { c/ } \\
\text { reconstrução }\end{array}$ & $1^{\circ} \mathrm{PO}$ & - $1^{\circ} \mathrm{PO}$ & $\begin{array}{l}\text { - orientações verbais + } \\
\text { manual + FST } \\
\text { - progressão gradual dos } \\
\text { exercícios } \\
\text { - reconstrução: início da FST } \\
2^{a} \text { semana }\end{array}$ & $\begin{array}{l}\text { - ADM completa: 4-6 } \\
\text { semanas } \\
\text { - FST: melhora da } \\
\text { qualidade de vida }\end{array}$ \\
\hline
\end{tabular}

$\mathrm{ADM}=$ amplitude de movimento; $\mathrm{MRM}=$ mastectomia radical modificada; $\mathrm{FST}=$ fisioterapia; pré-op.= pré-operatório; $\mathrm{PO}=$ pós-operatório; $\mathrm{AVD}=$ atividade de vida diária 
avaliação desses especialistas, pode-se constatar que houve um alto índice de concordância com relação à necessidade da realização de alongamentos da região cervical (Figura 1).

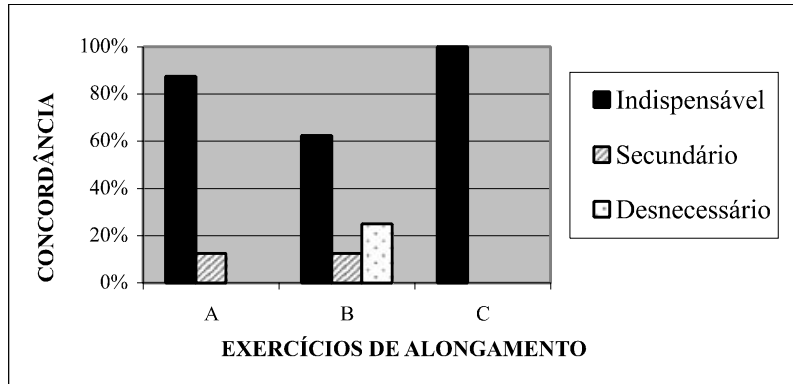

Figura 1. Índice de concordância entre os especialistas sobre os exercícios A, B e C para da região cervical propostos para o Programa de Reabilitação Precoce de mulheres mastectomizadas - SP, 2005

Em relação aos exercícios para movimento da cintura escapular, o resultado encontrado foi semelhante (Figura 2).

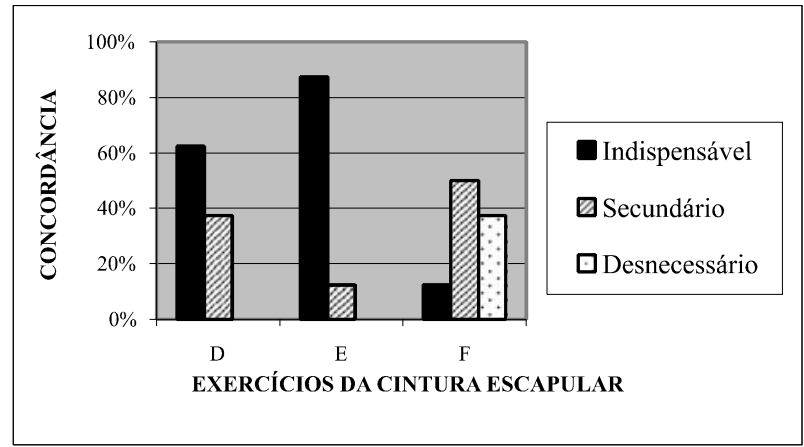

Figura 2. Índice de concordância entre os especialistas sobre os exercícios $D$, E e F para movimento da cintura escapular propostos para o Programa de Reabilitação Precoce de mulheres mastectomizadas - SP, 2005

No que se refere aos quatro exercícios de flexão dos ombros, dois deles apresentaram concordância de 100\% e dois, $80 \%$ (Figura 3).

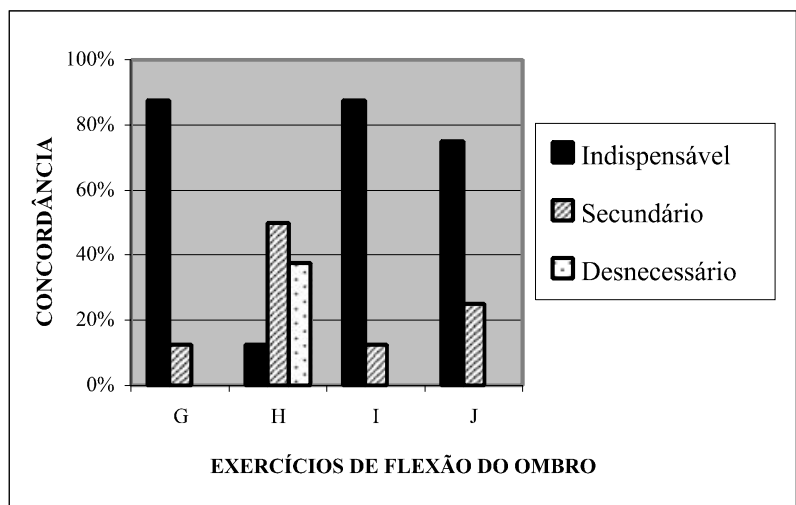

Figura 3. Índice de concordância entre os especialistas sobre os exercícios $G, H$, I e J para flexão dos ombros propostos para o Programa de Reabilitação Precoce de mulheres mastectomizadas SP, 2005
Os exercícios para extensão dos ombros foram os que apresentaram menor índice de concordância entre os especialistas, quanto à prioridade de inclusão no programa de exercícios (Figura 4).

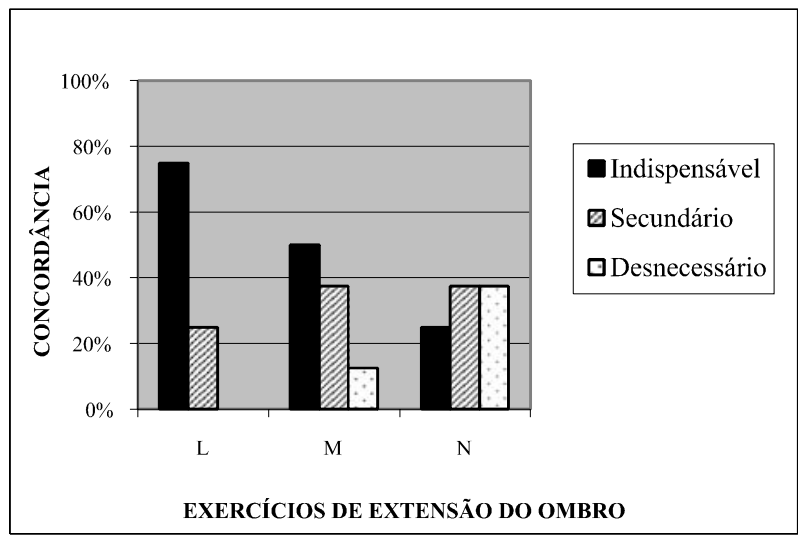

Figura 4. Índice de concordância entre os especialistas sobre os exercícios $L, M$ e $N$ para extensão dos ombros propostos para o Programa de Reabilitação Precoce de mulheres mastectomizadas SP, 2005

Nos exercícios para adução e abdução de ombro, houve alto índice de concordância entre os especialistas, sendo que apenas um dos cinco exercícios propostos foi considerado desnecessário (Figura 5).

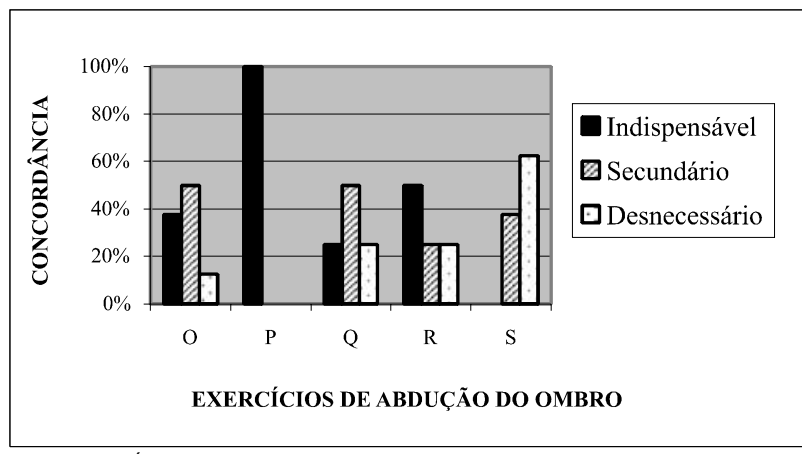

Figura 5. Índice de concordância entre os especialistas sobre os exercícios $O, P, Q, R$ e $S$ para abdução e adução dos ombros propostos para $\circ$ Programa de Reabilitação Precoce de mulheres mastectomizadas - SP, 2005

Com relação aos exercícios de fortalecimento, houve unanimidade entre os especialistas consultados de que os dois exercícios propostos não eram os mais indicados para o fortalecimento muscular e sugeriram a inclusão de outros exercícios, quer seja com uso de bolinhas juntamente com isometria do ombro a $90^{\circ}$, ou com uso de pesos leves, de 0,3 a $1 \mathrm{~kg}$, sendo esta modalidade de exercícios considerada prioritária para auxiliar a recuperação da $\mathrm{ADM}$, porém não em fase inicial de reabilitação (Figura 6). 


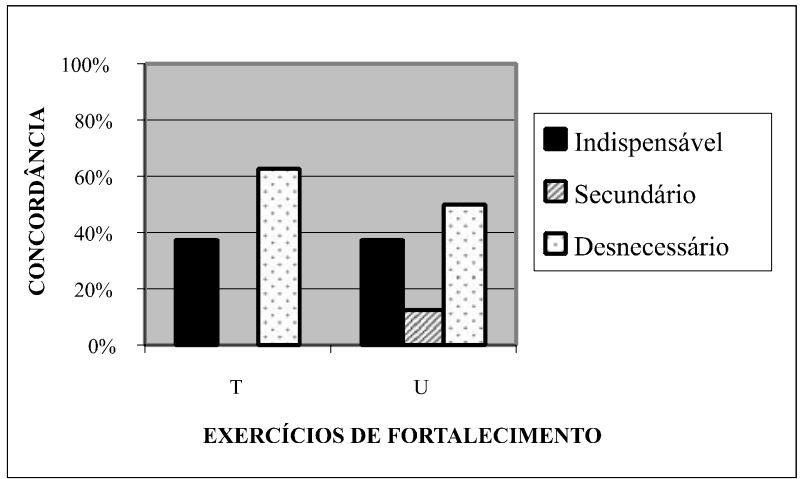

Figura 6. Índice de concordância entre os especialistas sobre os exercícios $T$ e $U$ para fortalecimento do antebraço propostos para o Programa de Reabilitação Precoce de mulheres mastectomizadas SP, 2005

Como a literatura ainda apresenta controvérsias quanto à realização de exercícios resistidos em mulheres mastectomizadas, e frente aos resultados obtidos com a avaliação dos especialistas, decidiu-se por não incluí-los.

Diante do exposto, o conjunto de exercícios selecionados a partir do consenso dos especialistas é o descrito a seguir:

- Alongamento e relaxamento da região cervical e da cintura escapular - exercícios A, C e E.

- Flexão dos ombros - exercícios G, I e J.

- Extensão dos ombros - exercício L.

- Adução e abdução dos ombros - exercício P.

Além desses oito exercícios, decidiu-se incluir também o F, frente à experiência com essa população, por já constar no manual utilizado anteriormente e por ser considerado eficiente no ganho de ADM da articulação do ombro ${ }^{16-17}$.

Esses exercícios compóem o novo manual do Programa Precoce de Exercícios para Reabilitação Funcional de Mulheres Mastectomizadas, que está sendo implementado no Setor de Oncomastologia da Disciplina de Mastologia da UNIFESP, desde agosto de 2006 (Anexo 2).

\section{DISCUSSÃO}

A revisão de literatura realizada mostrou que existem poucos trabalhos sobre Programas de Exercícios em pósoperatório de Mastologia com enfoque específico na recuperação da ADM. O aspecto abordado com maior freqüência é o relativo às complicações decorrentes dos tratamentos cirúrgico e radioterápico da neoplasia, principalmente o linfedema ${ }^{9,13,14}$.

Embora se reconheça a importância de programas para prevenção e tratamento do linfedema, deve-se dar ênfase à recuperação da $\mathrm{ADM}$ por meio de exercícios realizados precocemente como uma forma de contribuir para prevenção dessa complicação ${ }^{9,13,14}$.
Outro aspecto que chama a atenção é o reduzido número de estudos controlados randomizados relacionados à avaliação de programas de exercícios para recuperação da ADM. Dos 12 artigos identificados, apenas cinco eram randomizados, dos quais quatro apresentavam comparaçôes de tratamento com início precoce e tardio ${ }^{2,11,14-15}$, e um comparando exercícios com ADM livre versus ADM limitada a $90^{\circ}$.

Embora, na literatura consultada, haja consenso sobre a importância de um programa de exercícios pósoperatório com início precoce, com realização diária dos exercícios, de forma gradual e progressiva, respeitando os limites individuais das pacientes, há grande variabilidade entre os tipos de exercícios indicados, o número de repetições, e a duração total do programa.

No que diz respeito aos exercícios citados nos programas, houve unanimidade na indicação dos movimentos de flexão e abdução do ombro, sendo que alguns deles incluíam também exercícios de alongamento e relaxamento da região cervical, visto que a cirurgia, além de ocasionar a diminuição da ADM, pode levar a alterações posturais ${ }^{2-7,13,14}$.

Os exercícios classificados pelos especialistas como indispensáveis também incluíam essencialmente esses movimentos, fato observado pela seleção de três exercícios para alongamento da região cervical, dois para flexão e dois para abdução do ombro.

A inclusão de exercícios para extensão do ombro, além da linha média do corpo foi citada em apenas três $\operatorname{artigos}^{3,14,11}$. No entanto, na avaliação dos especialistas consultados, esses exercícios foram considerados indispensáveis na fase precoce de um programa de reabilitação. Uma possível explicação pode ser a necessidade desse movimento para a realização independente de algumas atividades de vida diária (AVD), como abotoar o sutiã, fechar o zíper de saias e vestidos etc.

Os exercícios para rotação interna e externa citados em quatro artigos ${ }^{3,4,5,6}$ não foram incluídos no conjunto de exercícios apresentados aos especialistas por considerar que, com a recuperação funcional da flexão e abdução do ombro, obtêm-se uma ADM que possibilita a realização das AVD de forma independente. Essa decisão foi tomada com base na revisão bibliográfica realizada, na qual em alguns estudos encontrados foram avaliadas somente a flexão e a abdução, e obteve-se ADM funcional compatível com as necessidades das pacientes ${ }^{5,7}$.

No que se refere à efetividade de um programa de exercícios com o objetivo de recuperar a ADM do ombro, verificou-se que os estudos consideram eficientes programas cujo resultado alcance a recuperação total ou parcial, com um déficit de $20^{\circ}$ a $30^{\circ}$ da ADM do ombro, considerando-se as medidas no pré-operatório e no final do programa $a^{2,13,18}$. 
O outro aspecto a ser destacado é a falta de consenso sobre a inclusão de exercícios de resistência ou fortalecimento muscular num programa de reabilitação, principalmente para mulheres submetidas à cirurgia, seja ela quadrantectomia ou mastectomia, associada ao esvaziamento axilar, para as quais é dada a orientação de não carregar pesos, a fim de evitar possíveis complicaçôes como deiscência, linfedema ou dor ${ }^{19,20}$. Quando citados, a indicação é de que sejam realizados, em fase mais tardia da reabilitação, por meio de exercícios isométricos ${ }^{14}$, com bolinha ${ }^{3}$, ou com uso de bandas elásticas ou resistência leve ${ }^{5,12}$. Em concordância com essa orientação, a inclusão desses exercícios deve ocorrer somente após a recuperação da ADM, num programa de seguimento de longo prazo.

Mesmo após a recuperação da ADM, é importante manter a realização de exercícios para manutenção da mesma, uma vez que pode haver diminuição e comprometimento da função, quer seja por redução das atividades, ou pela realização de tratamentos adjuvantes, como radioterapia e quimioterapia ${ }^{21}$.

Contudo, quando se propõe um seguimento por longo prazo, tem que se pensar em estratégias que promovam a adesão das pacientes, pois o número de desistências costuma ser elevado, acima de 50\% nos países em desenvolvimento, nos tratamentos de doenças crônicas ${ }^{22}$.

Sabe-se que a adesão à realização de exercícios em períodos críticos, como no tratamento do câncer, é um fator que pode ser otimizado com a utilização de algumas estratégias, como por exemplo: explicação detalhada do tipo de exercício a ser realizado, relacionando-o com as atividades de vida diária como forma de encorajamento e motivação, reorientações periódicas para adaptação dos exercícios às capacidades individuais e a funcionalidade que se deseja alcançar, bem como inclusão da família no processo de reabilitação ${ }^{23}$.

A partir dessas considerações sobre adesão a tratamentos prolongados, julga-se que um programa de exercícios, composto por um grande número dos mesmos, também pode dificultar a adesão das pacientes. Por esse motivo, considera-se que a seleção criteriosa de um número reduzido de exercícios, porém essenciais, para promover a funcionalidade do ombro, pode ser efetivo para a recuperação da ADM dessas mulheres.

Destaca-se que os nove exercícios selecionados para o programa proposto para reabilitação funcional das mulheres atendidas no setor de Oncomastologia da UNIFESP são aqueles que, além de terem sido considerados indispensáveis pelos especialistas consultados, incluem os movimentos preconizados pela literatura para essa finalidade. O desafio que se apresenta é testar sua efetividade por meio da avaliação do programa, que está em andamento no setor acima referido.

\section{CONSIDERAÇÕES FINAIS}

A revisão de literatura realizada permitiu a identificação de 12 estudos sobre exercícios pósoperatórios, a partir dos quais foi selecionado um conjunto de 20 exercícios, englobando os movimentos de flexão, extensão, abdução e adução do ombro. Destaca-se que, deste conjunto, nove foram considerados indispensáveis em uma fase precoce de reabilitação funcional pelos especialistas consultados.

O resultado desse estudo contribuiu para a reformulação do manual para Reabilitação Funcional Precoce de Mulheres Mastectomizadas do Setor de Oncomastologia da Disciplina de Mastologia da UNIFESP, o qual, ao mesmo tempo em que atende aos requisitos da reabilitação do movimento, constitui um recurso importante para suprir as necessidades das usuárias do serviço onde o programa será implementado.

Declaração de conflito de interesse: nada a declarar. 


\section{ANEXOS}

Anexo 1. Questionário com os exercícios submetidos à avaliação por especialistas

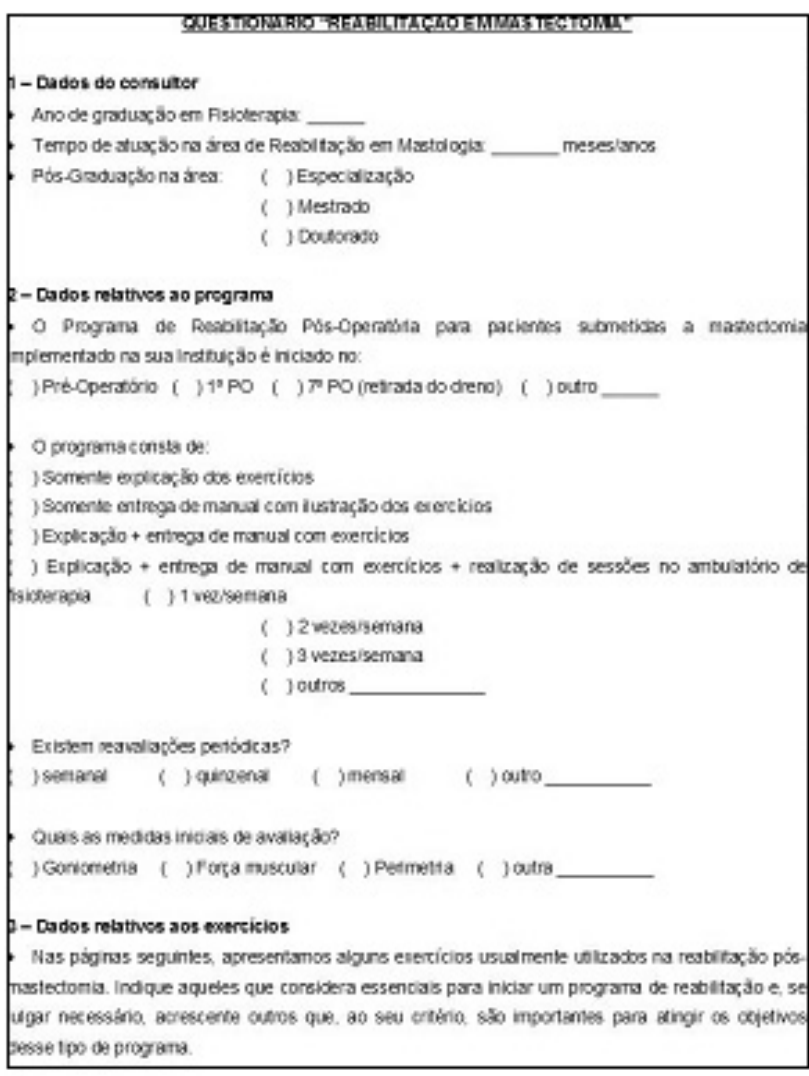

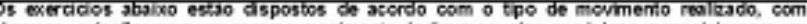

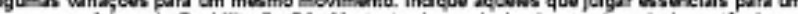

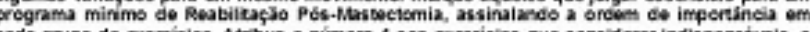
Fada grupo de exercicios. Atribua o numero 1 acs exercicios que sonsiderar indispensiveis, 0 himero 2 ass secundirios, eo nimere 3 ass que julgar cesnecessinios.

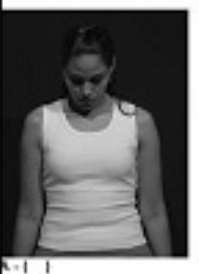
I - ALONGAMENTOS DA REGIÁO CERVICAL
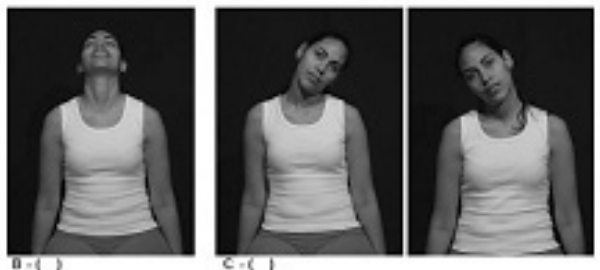

1 - MONIENTTOS DA CINTURA ESCAPULAR
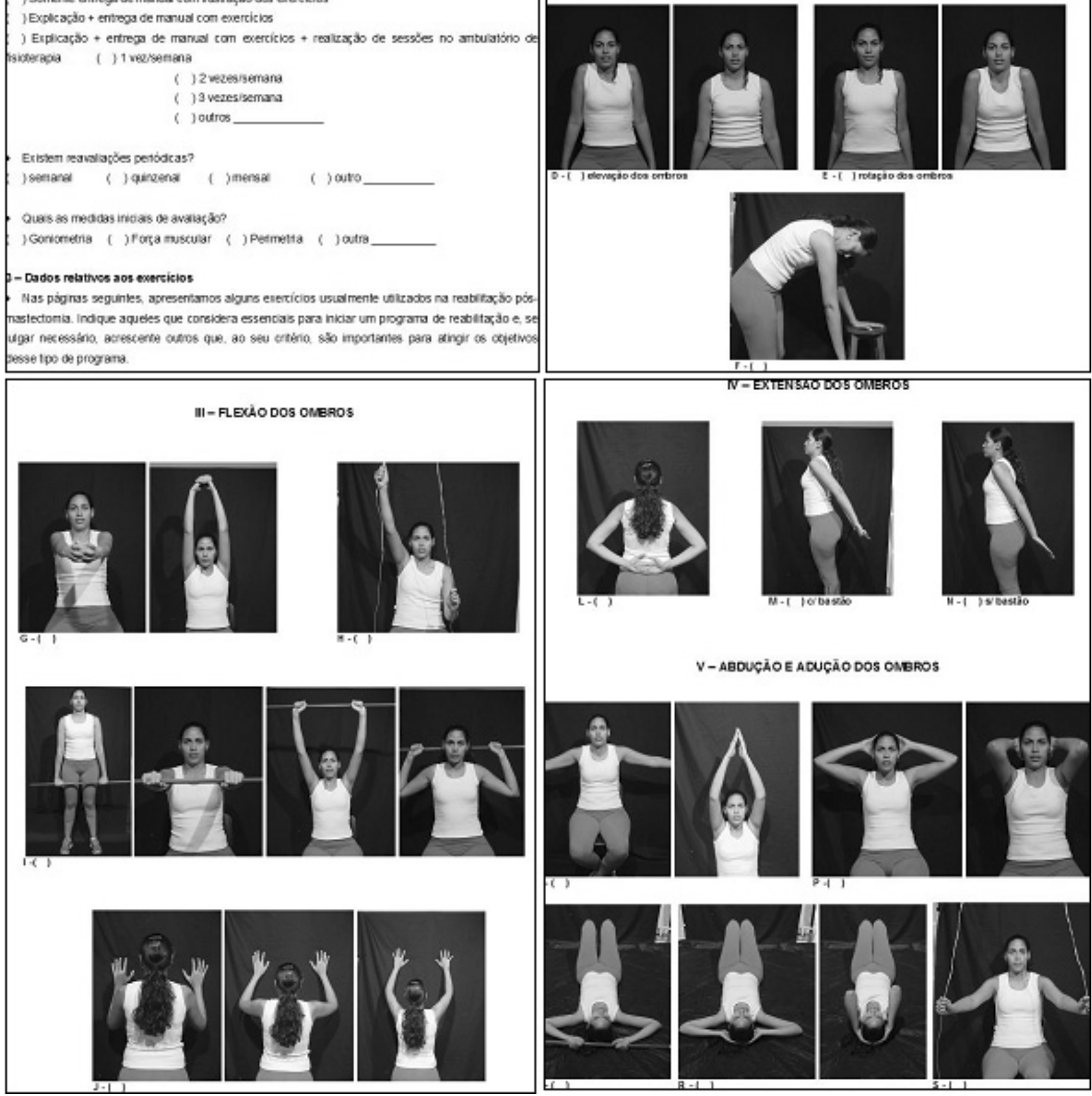

TV-EXTENSAODOSOMEROS

$\mathrm{L}-\mathrm{s}$
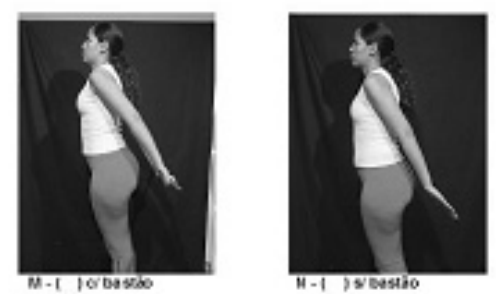

V-ABDUÇÃOE ACUÇĀO DOS OMBROS
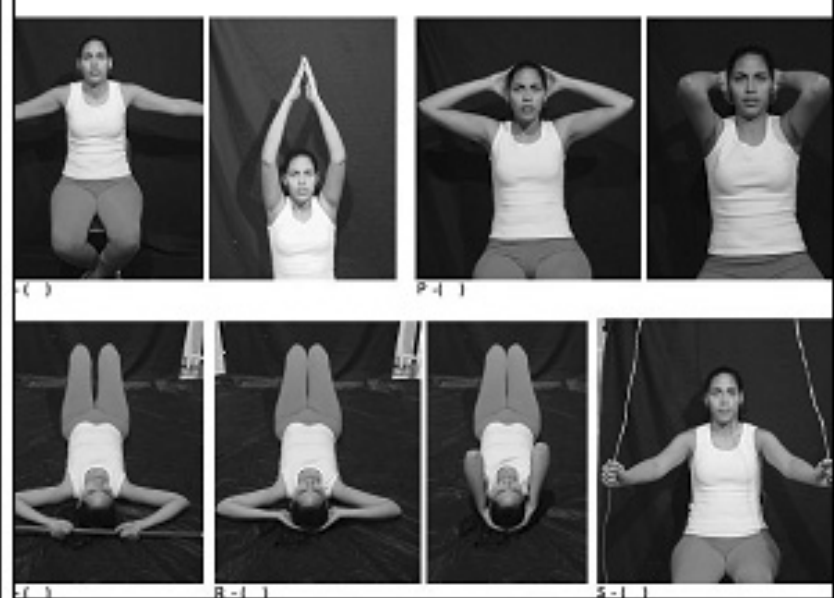

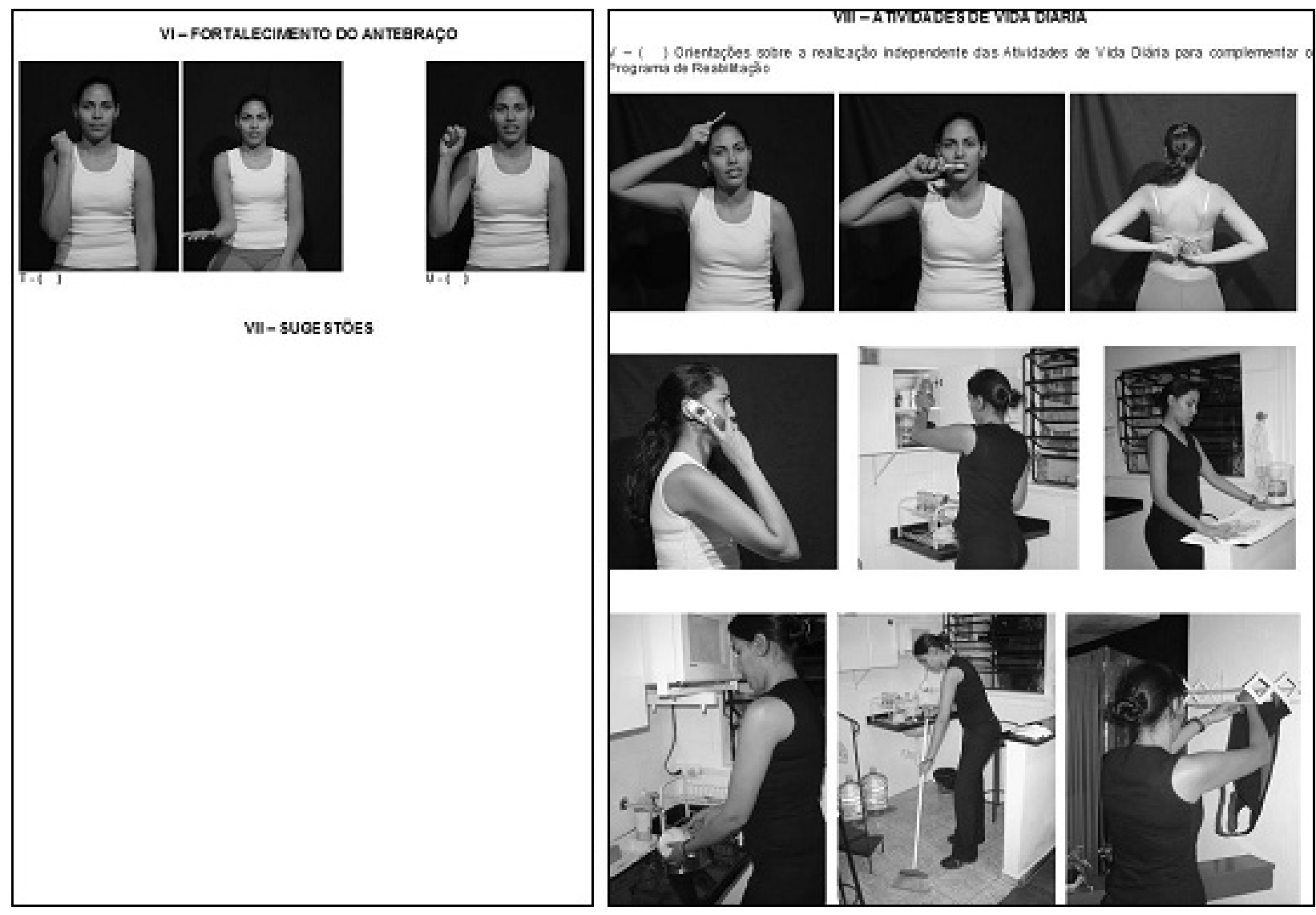
Anexo 2. Manual do Programa Precoce de Exercícios para Mulheres Mastectomizadas - Setor de Oncomastologia, Disciplina de Mastologia, UNIFESP, 2006

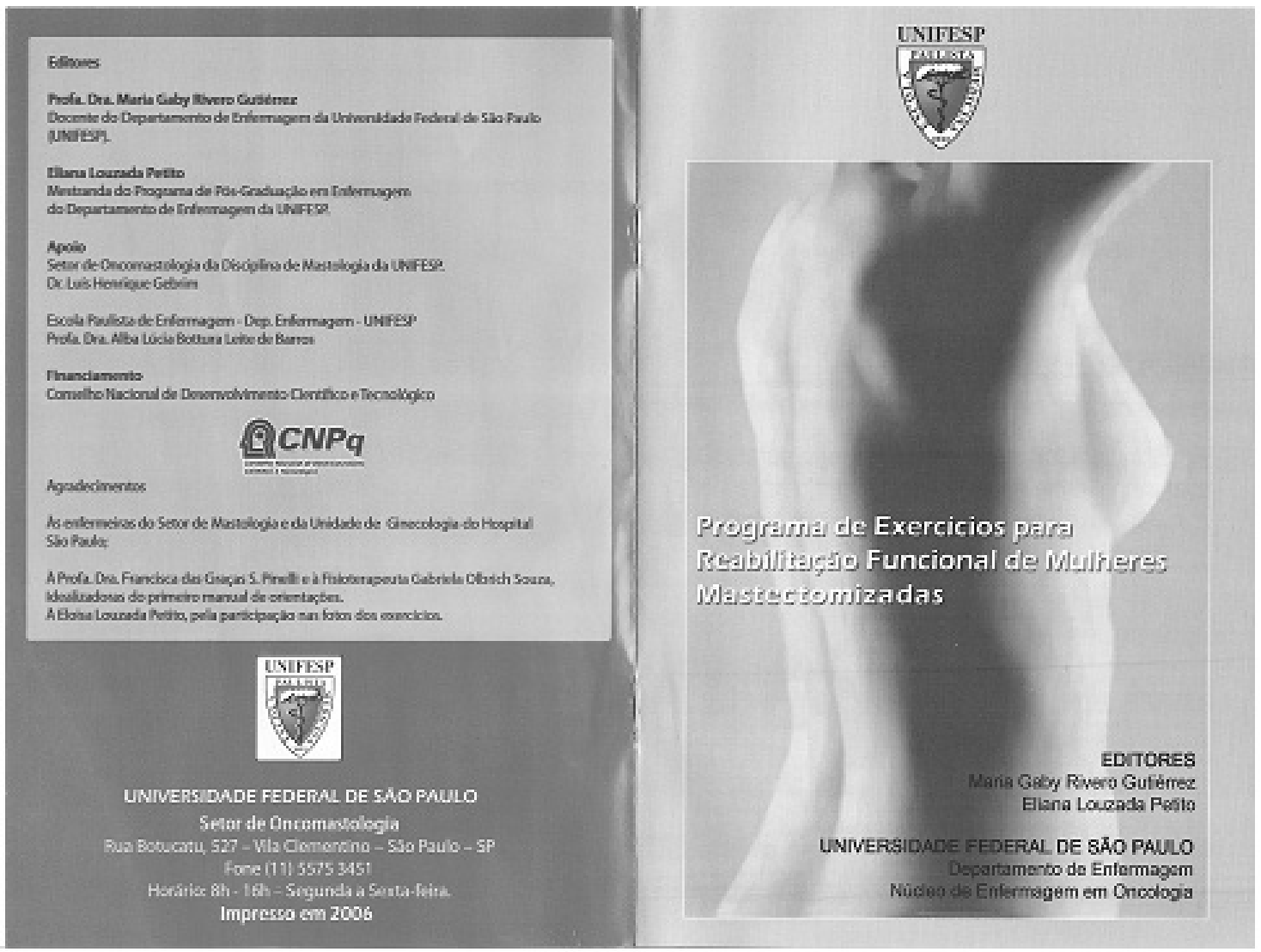

\section{LEMBRETES IMPORTANTES}

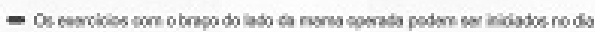

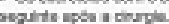

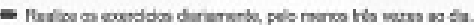

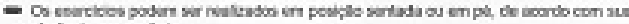
purkethoia ent confosts

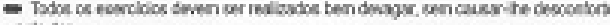
pele dee

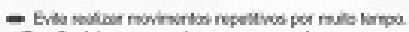

Tits

\section{Prezada Cliente}

Este manual foi criado especialmente para você, com a finalidade de oferecer-the informaçöes que a ajudem na realizaçăo dos exercicios e das atividades de vida diária, após a cirurgia.

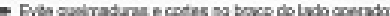

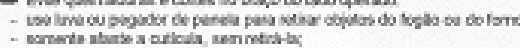

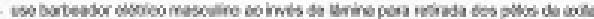

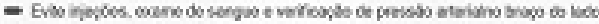
sperndo.

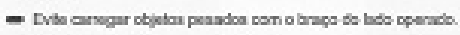

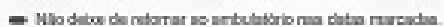


EXERCICIOS DO $1^{\circ}$ POS-OPERATORIO ATEA RETIRADA DO DRENO
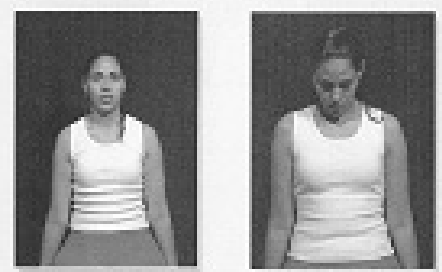

1

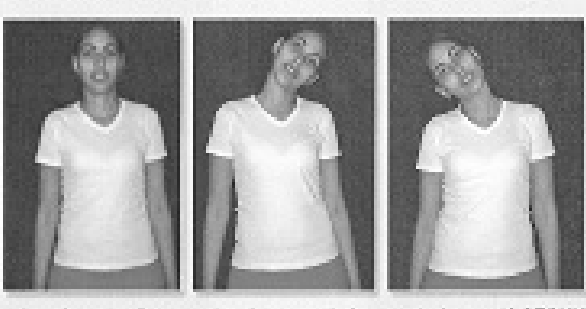

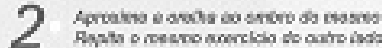
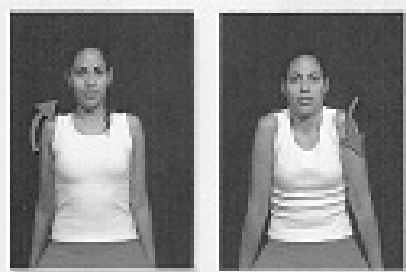

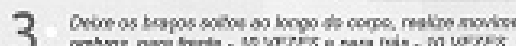

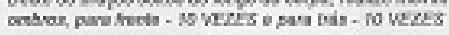

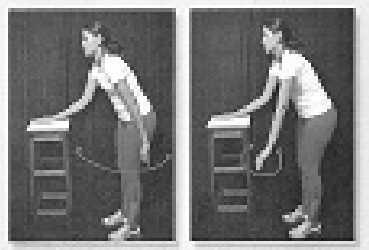

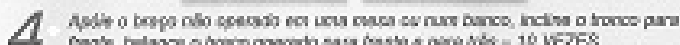

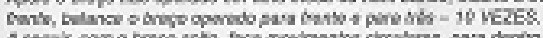

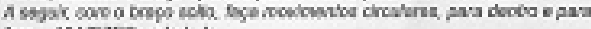
bava - N vezes corle ivats.
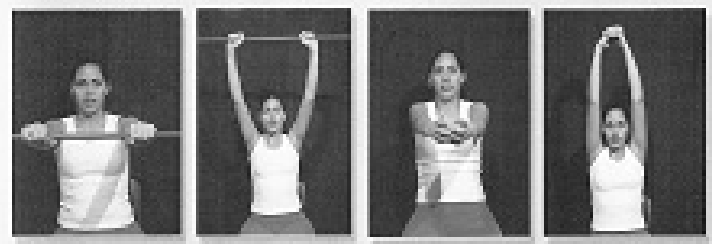

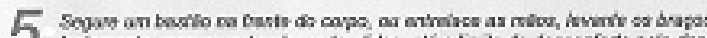

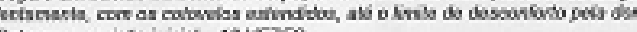
Mabarne a pasplo hieid - 10 VEZES

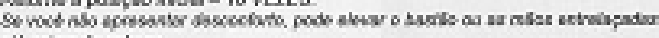
at actere da cabopa

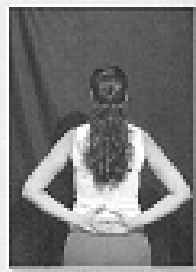

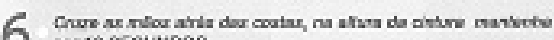
por 10 sebunoos

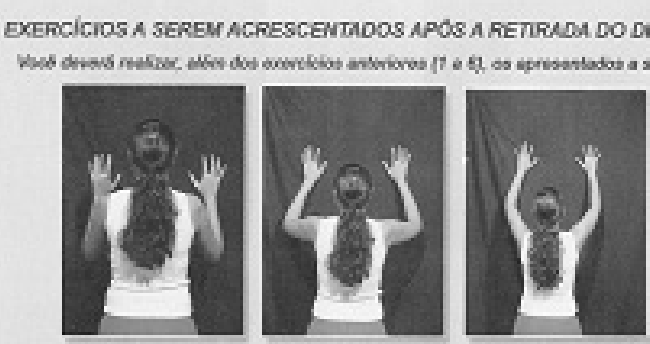

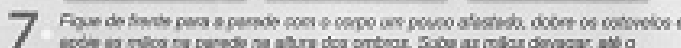

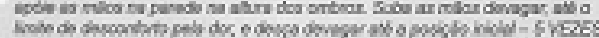
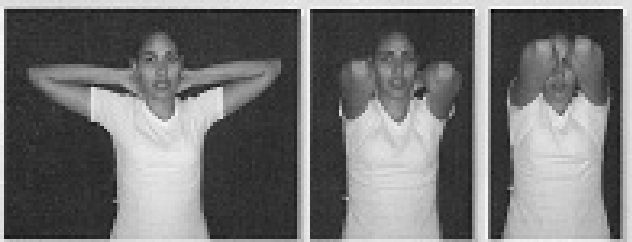

8

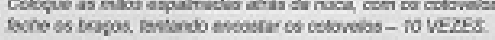
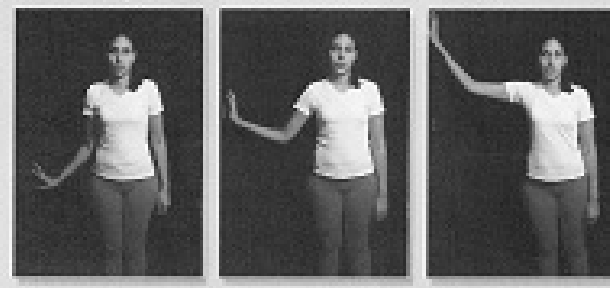

9

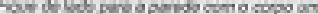

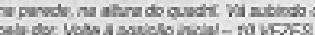

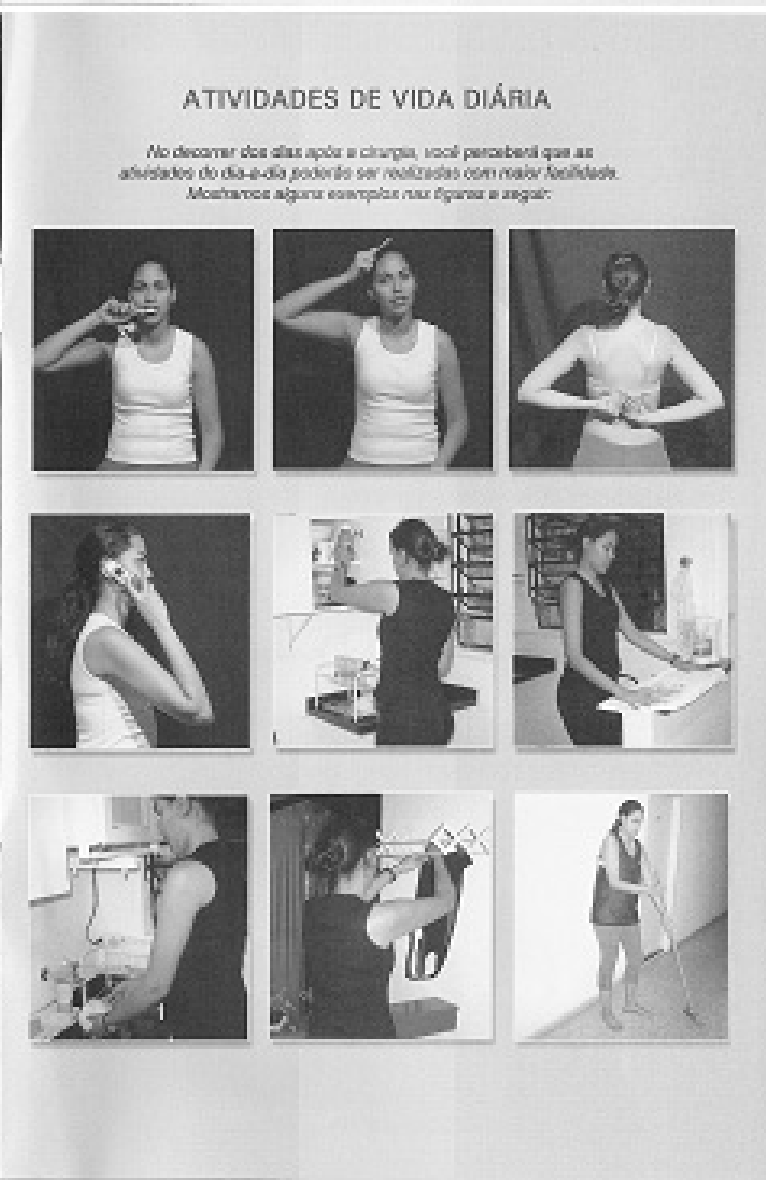




\section{REFERÊNCIAS}

1. Sasaki T, Lamari NM. Reabilitação funcional precoce pós mastectomia. HB Científica. 1997;4(2):121-7.

2. Box RC, Reul-Hirche HM, Bullock-Saxton JE, Furnival CM. Shoulder movement after breast cancer surgery: results of a randomized controlled study of postoperative physiotherapy. Breast Cancer Res Treat. 2002;75(1):35-50.

3. Bruno AA, Masiero D, Secco MFM, Wasserstein S, Chamlian TR. Padronizaçẫo de conduta na reabilitação póscâncer de mama. J Bras Med. 2000;78(5):86-92.

4. Marimoto T, Tamura A, Ichihara T, Minakawa T, Kuwamura Y, Miki M, et al. Evaluation of a new rehabilitation program for postoperative patients with breast cancer. Nurs Health Sci. 2003;5(4):275-82.

5. McAnaw MB, Harris KW. The hole of physical therapy in the rehabilitation of patients with mastectomy and breast reconstruction. Breast Dis. 2002;16:163-74.

6. Silva MPP, Derchain SFM, Rezende L, Cabello C, Martinez EZ. Movimento do ombro após cirurgia por carcinoma invasor da mama: estudo randomizado prospectivo controlado de exercícios livres versus limitados a $90^{\circ}$ no pósoperatório. Rev Bras Ginecol Obstet. 2004;26(2):125-30.

7. Pereira AMA, Vieira EORY, Alcântara PSM. Avaliação de protocolo de fisioterapia aplicado a pacientes mastectomizadas a Madden. Rev Bras Cancerol. 2005;51(2):143-8.

8. Prado MAS, Mamede, MV, Almeida AM, Clapis MJ. A prática da atividade física em mulheres submetidas à cirurgia por câncer de mama: percepção de barreiras e benefícios. Rev Latinoam Enferm. 2004;12(3):494-502.

9. Magaldi CM, Barros AC, Magaldi FM, Mantese JC, Pinotti, JA. Avaliação da morbidade e funcionalidade do membro superior em mulheres submetidas à linfadenectomia axilar total e biópsia de linfonodo sentinela por câncer de mama. Rev Bras Mastol. 2005;15(1)1:9-14.

10. Baraúna MA, Canto RST, Schulz E, Silva RAV, Silva CDC, Veras MTS, et al. Avaliação da amplitude de movimento do ombro em mulheres mastectomizadas pela biofotogrametria computadorizada. Rev Bras Cancerol. 2004;50(1):27-31.

11. Lauridsen MC, Christiansen P, Hessov IB. The effect of physiotherapy in shoulder function in patients surgically treated for breast cancer: a randomized study. Acta Oncol. 2005;44(5):449-57.
12. Na YO, Lee JS, Park JS, Kang SW, Lee HD, Koo JY. Early rehabilitation program in postmastectomy patients: a prospective clinical trial. Yonsei Med J. 1999;40(1):1-8.

13. Bergmann A, Ribeiro MJP, Pedrosa E, Nogueira EA, Oliveira ACG. Fisioterapia em Mastologia: rotinas do Hospital do Câncer III/INCA. Rev Bras Cancerol. 2006;52(1):97-109.

14. Rezende LF, Franco RL, Rezende MF, Beletti PO, Moraes SS, Gurgel MSC. Two exercises schemes in postoperative breast cancer: comparison of effects on shoulder movement and lymphatic disturbance. Tumori. 2006;92(1):55-61.

15. Leal TO, Cardoso KQ, Kalif SK, Almeida FB, Fontelles MJ. A fisioterapia na disfunção motora do membro superior pós-mastectomia radical modificada. Rev Para Med. 2005;19(2):47-52.

16. Silva IA, Lima PP. A biocinese do ombro e suas patologias. [monografia na Internet]. Belém (PA): Universidade da Amazônia; [citado em 2007 jan 25]. Disponível em http:/ /www.nead.unama.br/site/bibdigital/pdf/artigos_revistas/ 87.pdf.

17. Rezende LF, Beletti PO, Franco RL, Moraes SS, Gurgel MSC. Exercícios livres versus direcionados nas complicaçōes pós-operatórias de câncer de mama. Rev Assoc Med Bras. 2006;52(1):37-42

18. Rietman JS, Dijkstra PU, Hoekstra HJ, Eisma WH, Szabo BG, GroothoffJW, et al. Late morbidity after treatment of breast cancer in relation to daily activities and quality of life: a systematic review. Eur J Surg Oncol. 2003;29(3):229-38.

19. Magaldi CM, Pinotti JA. Atenção fisioterapêutica após cirurgia para o câncer de mama. In: Piato S, Piato JRM, editores. Doenças da mama. Rio de Janeiro: Revinter; 2006. p. 230-9.

20. Smith CM. Postmastectomy training. IDEA Personal Trainer. 2001 Oct: 39-44.

21. Kim CJ, Kang DH, Smith BA, Landers KA. Cardiopulmonary responses and adherence to exercise in women with breast cancer undergoing adjuvant therapy. Cancer Nurs. 2006;29(2):156-65.

22. Poor adherence to long-term treatment of chronic diseases is a worldwide problem. Rev Panam Salud Publica. 2003;14(3):218-21.

23. Coleman EA, Hall-Barrow J, Coon S, Stewart CB. Facilitating exercise adherence for patients with multiple myeloma. Clin J Oncol Nurs. 2003;7(5):529-34. 


\section{Abstract}

Background: The benefits of early functional rehabilitation in women submitted to breast cancer surgery are widely known, but there is no consensus on the best exercises or their periodicity and duration. Objective: The objectives were to identify and select a set of exercises for a postoperative rehabilitation program aimed at functional recovery of the ipsilateral limb and to validate this program with experts. Methods: Review of scientific articles on the effectiveness of functional rehabilitation programs for shoulder range of movement following breast cancer surgery, published from 1997 to 2006 in the MEDLINE, LILACS, and PUBMED databases. Selection of a set of exercises for the recovery of shoulder range of movement and its evaluation by rehabilitation experts. Results: Of the 21 articles found, 12 reported on testing or standardization of procedures for post-mastectomy physical rehabilitation, which constituted the basis for selecting the 20 exercises submitted to expert evaluation. The experts considered nine of these exercises indispensable, with a concordance rate greater than or equal to $75 \%$. The exercises that achieved the highest concordance rates were cervical stretching, shoulder flexion, extension, and abduction. Conclusion: The study findings contributed greatly to the reorganization of the existing manual at the Breast Cancer Service of the Federal University in São Paulo (UNIFESP).

Key words: Exercise; Mastectomy; Breast neoplasms; Physical therapy (specialty); Oncology nursing 